\title{
Effect of Particle Size on Skin Permeation and Retention of Piroxicam in Aqueous Suspension
}

\author{
Kunikazu Moribe, ${ }^{*, a}$ Mizuho Shibata, ${ }^{a}$ Takayuki Furuishi, ${ }^{b}$ Kenjirou Higashi, ${ }^{a}$ Kazuo Tomono, ${ }^{b}$ and

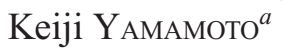 \\ ${ }^{a}$ Graduate School of Pharmaceutical Sciences, Chiba University; 1-8-1 Inohana, Chuo-ku, Chiba 260-8675, Japan: and \\ ${ }^{b}$ Research Unit of Pharmaceutics, School of Pharmacy, Nihon University; 7-7-1 Narashinodai, Funabashi, Chiba \\ 274-8555, Japan. Received March 16, 2010; accepted May 1, 2010; published online May 13, 2010
}

\begin{abstract}
The effects of particle size on the skin permeation and retention of piroxicam (PXC) in an aqueous suspension were investigated. PXC particles of about $23 \mathrm{~nm}, 173 \mathrm{~nm}$, and $2.1 \mu \mathrm{m}$ in size were prepared by the cogrinding of PXC/polyvinylpyrrolidone (PVP) K12/sodium dodecyl sulfate physical mixture (mean particle size, $9.6 \mu \mathrm{m})$ using a vibrational rod and ball mills. Particles were stable after storage in $0.1 \mathrm{M}$ acetate buffer (pH 4.5) for $24 \mathrm{~h}$. The amount of PXC that permeated and was retained in hairless mouse skin increased with the reduction of particle size up to $23 \mathrm{~nm}$. Amorphous PXC was formed when PXC was coground with PVP, though the amorphous formation did not affect the amount of PXC permeated. Reduction of particle size to less than $50 \mathrm{~nm}$, and the subsequent increase in surface area of PXC nanocrystals appeared to affect skin permeation and retention behavior.
\end{abstract}

Key words skin permeation; retention; particle size; cogrinding; poorly water-soluble drug; aqueous suspension

Reduction in particle size of hydrophobic drugs has attracted interest as a way to improve their dissolution and absorption properties. ${ }^{1-4)}$ Grinding is one of the methods used to reduce the size of drug crystals. Cogrinding of a hydrophobic drug with a hydrophilic polymer and surfactant appears to be an effective method for the preparation of drug nanoparticles and stable nanosuspensions. ${ }^{5-8)}$ Using a drug/ polyvinylpyrrolidone K17 (PVP K17)/sodium dodecyl sulfate (SDS) ternary ground mixture (GM) at a weight ratio of $1 / 3 / 1$, more than 20 kinds of drugs have been effectively reduced to nano-order particle size. ${ }^{6)}$ When a probucol/PVP K12/SDS GM suspension was orally administered to rats, plasma concentration increased as particle size decreased. ${ }^{8)}$ In particular, an increase in plasma concentration was observed when a GM suspension with a particle size of about $28 \mathrm{~nm}$ was used.

Drug nanoparticles are useful not only for use in oral administration but also for use in intravenous, subcutaneous, and transdermal administration. Transdermal administration is a promising candidate for drug delivery because of its association with the following advantages: avoidance of a hepatic first-pass effect, reduction in the frequency of drug administration, and improved patient compliance. The effectiveness of transdermal drug delivery has been demonstrated in several studies. Standard practices to improving skin permeation and retention of drugs include selection and modification of a drug molecule, ${ }^{9-11)}$ drug complex formation with excipients such as cyclodextrin, ${ }^{12-14)}$ and addition of a permeation enhancer. ${ }^{15-17)}$ Recently, the use of drug nanoparticles has been shown to improve drug delivery to the skin. ${ }^{18-24)}$ Surface area enhancement due to the size reduction of drug crystals or their amorphous formation may affect skin permeation behavior. Nano-sized drug may also contribute to the enhanced retention, which is applicable for the topical administration. However, the effect of drug particle size on skin permeation and retention behavior has not been precisely investigated.

The purpose of this study is to determine the effect of par- ticle size of a hydrophobic drug in aqueous suspension on skin permeation and retention. Piroxicam (PXC) was used as a model drug, and reduction of crystal size was performed by ternary cogrinding with PVP K12 and SDS. After dispersing the coground sample in an aqueous medium, the PXC suspension was used to determine skin permeation and retention behavior.

\section{Experimental}

Materials PXC was purchased from Wako Pure Chemical Industries Ltd. (Osaka, Japan). PVP K12 (molecular weight [Mw], 2500), a water-soluble polymer, and SDS, an anionic surfactant, were obtained from BASF Japan Ltd. (Tokyo, Japan) and Wako Pure Chemical Industries Ltd., respectively. All other chemicals used were of reagent grade.

Preparation of Physical Mixture (PM) PXC (0.5 g), PVP K12 (1.5 g), and SDS $(0.5 \mathrm{~g})$ at a weight ratio of $1: 3: 1$ were physically mixed in a glass vial for 5 min using a vortex mixer.

Preparation of Ground Mixture (GM) Three PXC/PVP K12/SDS ternary GMs with different particle size were prepared. For GM1, the PM was ground using a planetary ball mill (PM 100, Retsch Co., Ltd., Haan, Germany) under the following conditions: volume of pot, $50 \mathrm{ml}$; material of pot, alumina; balls, $\phi 20 \mathrm{~mm} \times 2$; rotation speed, $200 \mathrm{rpm}$; and rotation time, $180 \mathrm{~min}$. For GM2, the PM was ground using the planetary ball mill as follows: balls, $\phi 10 \mathrm{~mm} \times 10$; rotation speed, $400 \mathrm{rpm}$; and rotation time, $150 \mathrm{~min}$. For GM3, the PM was ground using a vibrational rod mill (TI-200, Heiko Seisakusho, Tokyo, Japan) for $30 \mathrm{~min}$.

Powder X-ray Diffraction (PXRD) Measurement PXRD measurements were performed using a Rigaku MiniFlex II diffractometer (Tokyo, Japan), under the following conditions: target, copper $(\mathrm{Cu})$; filter, nickel $(\mathrm{Ni})$; voltage, $30 \mathrm{kV}$; current, $15 \mathrm{~mA}$; scanning speed, $4 \% \mathrm{~min}$; and scanning angle, $3-35^{\circ}$

Particle Size Analysis The GM powder was dispersed in a $0.1 \mathrm{~m}$ acetate buffer (pH 4.5) and then sonicated for 2 min to form a colloidal suspension. Particle drug size was determined by the dynamic light scattering method using a Microtrac UPA (Nikkiso, Tokyo, Japan; measurement range, $0.003-6 \mu \mathrm{m})$ and by the light scattering method using a Microtrac FRA (Nikkiso, Tokyo, Japan; measurement range, $0.1-700 \mu \mathrm{m}$ ) at $25^{\circ} \mathrm{C}$.

Skin Permeation Studies All animal experiments were carried out in accordance with the guidelines of the Institutional Animal Care and Use Committee (School of Pharmacy, Nihon University, Chiba, Japan). The fullthickness dorsal skin of male Hos:HR-1 hairless mice (5-10 weeks old, Hoshino Laboratory Animals, Inc., Ibaragi, Japan), sacrificed by ether, was excised, and adherent fat and other visceral debris were removed from the undersurface. In vitro permeation studies were carried out with Franz diffu- 
sion cells (Vertical Diffusion Cell, Hanson Research Corp., CA, U.S.A.) at $32{ }^{\circ} \mathrm{C}$. The effective area of diffusion was $1.77 \mathrm{~cm}^{2}$, and the receiver cell volume was about $7.0 \mathrm{ml}$. The donor compartment was filled with $1.0 \mathrm{ml}$ of GM suspension containing $3.0 \mathrm{mg} / \mathrm{ml}$ of $\mathrm{PXC}$ in $0.1 \mathrm{M}$ acetate buffer $(\mathrm{pH} 4.5)$. The receiver cell was filled with phosphate buffered saline (PBS; pH 7.4) and stirred at $650 \mathrm{rpm}$ with a magnetic stirrer. The amount of permeated PXC was quantitated by collecting $1.0 \mathrm{ml}$ of samples at designated time intervals $(1,2,3,4,5,6,7,8,22,24 \mathrm{~h})$. The volume of receptor fluid withdrawn was replaced with PBS.

Extraction of PXC from Skin Samples Extraction of PXC from skin samples was carried out using a modified method reported by Okuryama et $a l^{25)}$ After the skin permeation experiment, a residual PXC sample was removed, and the skin surface was washed with methanol. Next, $1.13 \mathrm{~cm}^{2}$ of the skin was cut with a punch and homogenized in a methanol solution using a glass homogenizer at $1200 \mathrm{rpm}$ for $10 \mathrm{~min}$. The methanol solution was centrifuged at $3357 \times \boldsymbol{g}$ for $10 \mathrm{~min}$. The supernatant was diluted by mobile phase as described below.

Analytical Method The concentration of PXC in the sample was analyzed by HPLC. The HPLC system was constructed with a Model PU-2080 plus intelligent HPLC pump, a Model UV-2075 intelligent UV/VIS detector, and a Model AS-2055 plus intelligent sampler (all from Jasco Co., Tokyo, Japan). The analytical column, Capcell Pak C18, type UG120 (4.6 mm× $150 \mathrm{~mm}$ in diameter, particle size $5 \mathrm{~mm}$; Shiseido Co., Ltd., Tokyo, Japan), was used with a column oven (860-CO, Jasco Co.) at $40 \pm 1{ }^{\circ} \mathrm{C}$. The mobile phase consisted of acetonitrile and $4.0 \%$ acetic acid $(45: 55, \mathrm{v} / \mathrm{v})$ at a flow rate of $1.0 \mathrm{ml} / \mathrm{min}$. The column elute was monitored with UV wavelengths of $350 \mathrm{~nm}$.

Data Analysis The cumulative amount of PXC permeating the skin was plotted against time. Steady-state flux was calculated from the slope of the linear region of the time plot. The amount of PXC in the skin was calculated by conversion to the total area of skin.

\section{Results and Discussion}

Physicochemical Properties of PXC/PVP K12/SDS GM and Suspension To determine the effect of particle size on the permeation of PXC through the skin, PXC/PVP K12/SDS GMs with different particle sizes were prepared. The mean particle size of the PMs and GMs in $0.1 \mathrm{~m}$ acetate buffer ( $\mathrm{pH} 4.5)$ after storage at $25^{\circ} \mathrm{C}$ for $24 \mathrm{~h}$ are shown in Fig. 1. The solubility of PXC in the acetate buffer $(\mathrm{pH} 4.5)$ at $25^{\circ} \mathrm{C}$ was determined to be $27 \mu \mathrm{g} / \mathrm{ml}$, almost the same as its solubility in water. The mean particle size of the PM was $9.6 \mu \mathrm{m}$, similar to that of unprocessed PXC $(12.5 \mu \mathrm{m})$. However, the mean particle sizes of GM1, GM2, and GM3 were reduced to $2.1 \mu \mathrm{m}, 173 \mathrm{~nm}$, and $23 \mathrm{~nm}$, respectively. These results indicate that different grinding methods produce different particle sizes of PXC. Particle size did not change after storage for $24 \mathrm{~h}$, indicating that the formed particles are stable in aqueous solution.

The effect of different grinding conditions on molecular states of PXC in the GM powder was investigated by PXRD measurement (Fig. 2). When PXC was coground with PVP K12 at a molar ratio of $1: 3$, the PXRD showed a halo pattern. Crystalline PXC became amorphous by cogrinding with PVP K12 (Fig. 2d). In contrast, diffraction peaks originating from PXC crystals were observed in GM1 (Fig. 2f), GM2 (Fig. 2g), and GM3 (Fig. 2h). The peak intensity of GM2 and GM3 was apparently lower than that of GM1. Pongpeerapat et al. reported that the surface of probucol nanocrystals coground with PVP and SDS becomes amorphous through the intermolecular interaction of probucol with PVP. ${ }^{7)}$ In the PXC ternary system, the presence of amorphous PXC on the nanocrystalline surface and/or size reduction of PXC crystals to submicron-size order may contribute to the reduced diffraction peak intensity.

Effects of Particle Size on Skin Permeation and Reten- tion of PXC from the Ternary GM Suspension The effect of particle size on the amount of PXC that permeated hairless mouse skin is shown in Fig. 3a. The amount of PXC in GMs that permeated the skin was clearly higher than the permeation that occurred with PXC in the PM and in unprocessed PXC. The permeation parameters of PXC calculated from the graphs in Fig. 3 are shown in Table 1. Both the cumulative amount permeated for $8 \mathrm{~h}$ and the flux of GMs were significantly higher than those for the PM and unprocessed PXC. These results indicate that size reduction of
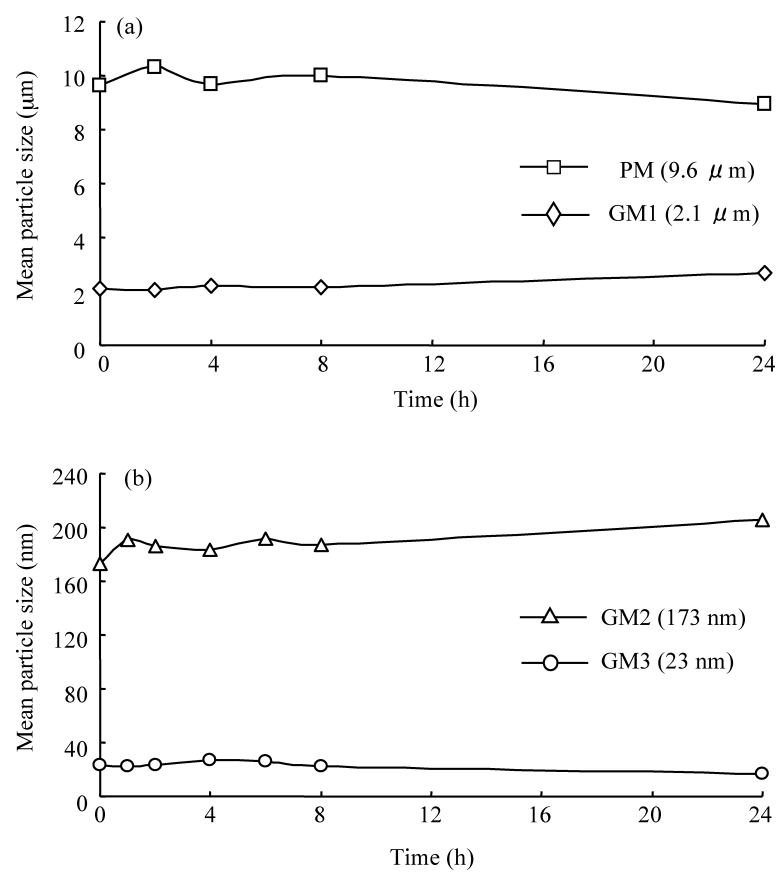

Fig. 1. Changes in Mean Particle Size of PXC/PVP K12/SDS Ternary PM and GMs in $0.1 \mathrm{M}$ Acetate Buffer ( $\mathrm{pH} 4.5$ )

Weight ratio of PXC/PVP K12/SDS was 1/3/1. Mean particle size was measured by (a) Microtrac HRA and (b) Microtrac UPA. Initial particle size of each sample is shown in parentheses.
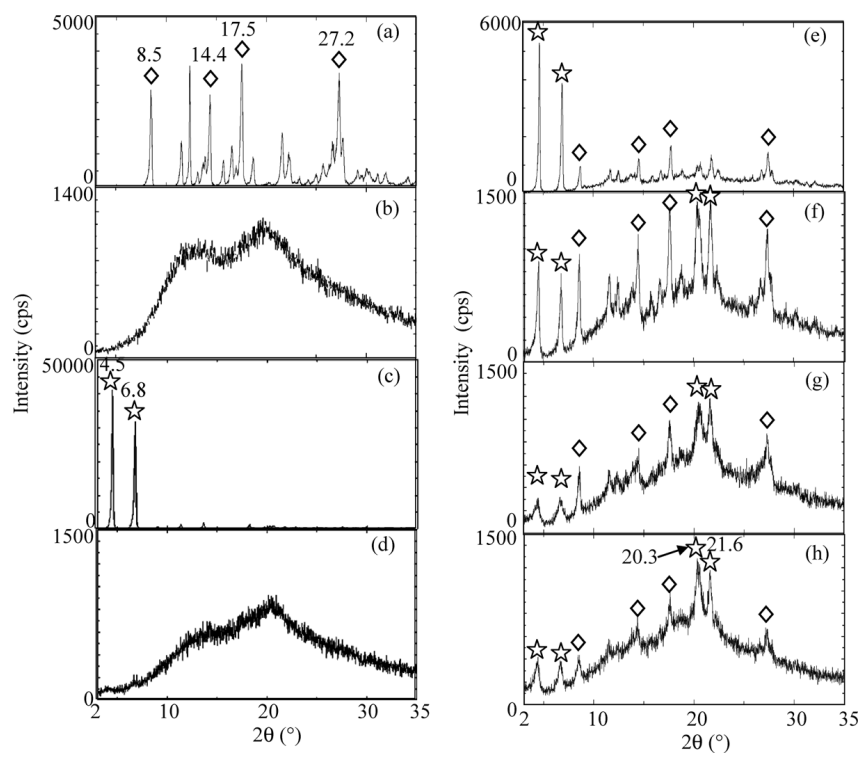

Fig. 2. PXRD Patterns of the PXC/PVP K12/SDS System

(a) Unprocessed PXC, (b) PVP K12, (c) SDS, (d) PXC/PVP K12 GM (1/3, w/w; 90-

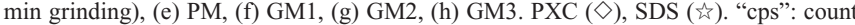
per second. 
PXC facilitates skin permeation. The effects of particle size on the skin retention of PXC in the PXC/PVP K12/SDS system are shown in Fig. 3b. The amounts of PXC in the skin were 2.3, 3.0, and 9.3 times higher than the amounts of unprocessed PXC in GM1, GM2, and GM3, respectively. Size reduction of the PXC particles contributed to enhanced skin retention.

The effects of particle size on skin permeation and retention have been reported in several studies. Shim et al. reported on the transdermal delivery of minoxidil with amphiphilic block copolymer nanoparticles using poly( $\varepsilon$-caprolactone)-block-poly(ethylene glycol). ${ }^{18)}$ They observed sizedependent drug permeation in hairy guinea pig skin; namely, the permeation of the minoxidil incorporated in 40-nm particles was higher in both the epidermal layer and the receptor solution than the permeation of minoxidil in 130-nm particles. However, because size-dependent permeation behavior
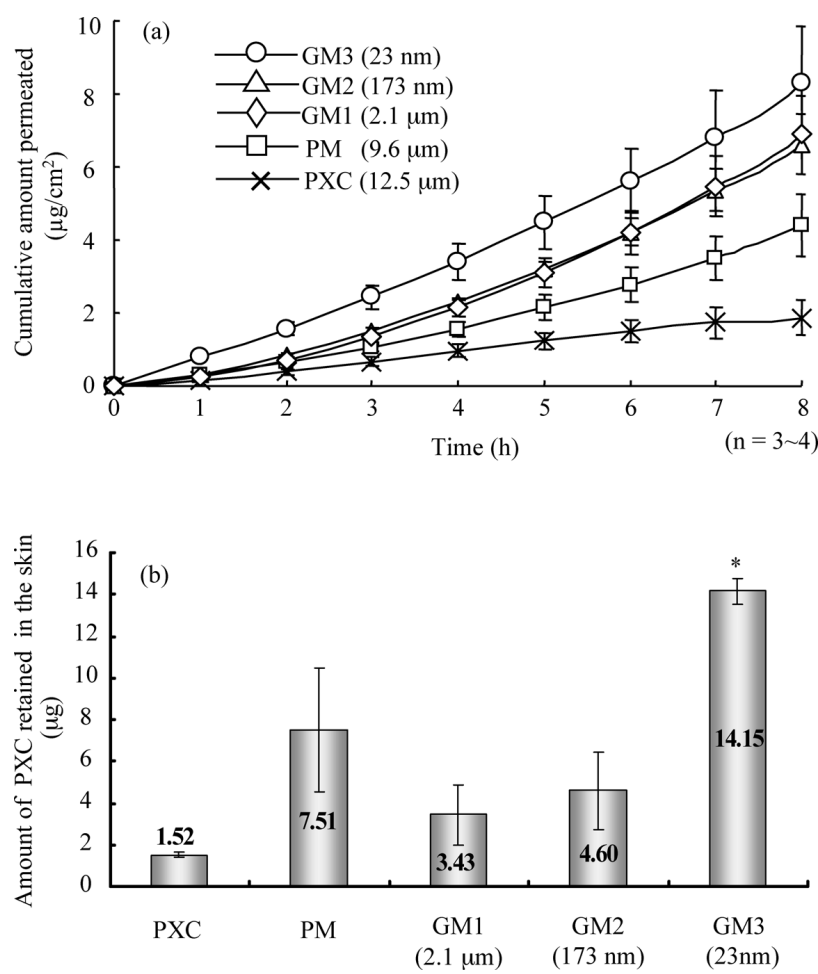

Fig. 3. Effect of Particle Size on Amount of (a) PXC Permeation and (b) Skin Retention

Each point represents the mean \pm S.D. of three to four experiments. $* p<0.01$ vs. PXC, PM, GM1, and GM2. Statistical analysis was performed by ANOVA.

Table 1. Skin Permeation Parameters of PXC Particles

\begin{tabular}{ccc}
\hline \hline $\begin{array}{c}\text { Sample } \\
(\text { size })\end{array}$ & $\begin{array}{c}\text { Cumulative amount permeated } \\
\text { for } 8 \mathrm{~h}\left(\mu \mathrm{g} / \mathrm{cm}^{2}\right)\end{array}$ & $\begin{array}{c}\text { Flux } \\
\left(\mu \mathrm{g} / \mathrm{cm}^{2} / \mathrm{h}\right)\end{array}$ \\
\hline PXC & $1.87 \pm 0.49$ & $0.25 \pm 0.06$ \\
PM & $4.39 \pm 0.86^{a)}$ & $0.58 \pm 0.13^{b)}$ \\
GM1 & $6.88 \pm 1.09^{b, c)}$ & $0.95 \pm 0.16^{b, c)}$ \\
$(2.1 \mu \mathrm{m})$ & $6.62 \pm 0.81^{b, d)}$ & $0.90 \pm 0.12^{b, c)}$ \\
GM2 & $8.31 \pm 1.56^{b, c)}$ & $1.07 \pm 0.21^{b, c)}$ \\
$(173 \mathrm{~nm})$ & & \\
\hline GM3 & & \\
\hline
\end{tabular}

Each value represents the mean \pm S.D. of $3-4$ experiments. $a$ ) $p<0.05 v s$. PXC, $b$ $p<0.01$ vs. PXC, c) $p<0.01$ vs. PM, $d) p<0.05$ vs. PM. Statistical analysis was performed by ANOVA. was not observed for hairless skin, the enhanced permeation mechanism was considered to be due to the permeation of solute-containing nanoparticles through shunt routes such as hair follicles. Similar permeation phenomena were also reported by Vogt et al. using particles of $1500 \mathrm{~nm}, 750 \mathrm{~nm}$, and $40 \mathrm{~nm}$ with fluorescence. ${ }^{19)}$ In that study, particle size reduction to $50 \mathrm{~nm}$ or smaller was found to be important for permeation enhancement.

In contrast, enhanced permeation by PXC nanoparticles was observed in the current study using hairless mouse skin, which has a limited number of hair follicles and other dermal appendages. Mortensen et al. described results of a permeation study of 30-nm quantum dots using fluorescent microscopy. ${ }^{24)}$ In experiments using hairless mice, quantum dots remained in the stratum corneum and did not reach the epidermis. These results suggest that the penetration of hairless mouse skin by nanoparticles is difficult even when particle size is less than $50 \mathrm{~nm}$. Thus, we speculated that the size reduction and the subsequent increase of surface area of PXC nanocrystals both contribute to the enhanced dissolution of the drug from the particle surface. These characteristics therefore may play an important role in the enhanced permeation and retention of PXC from the ternary GM suspension.

Effects of Amorphous PXC on Skin Permeation and Retention It has been reported that the amorphous form of drug in a matrix formulation contributes to improved skin permeation. ${ }^{26)}$ As shown in Fig. 2d, PXC became amorphous after it was coground with PVP K12. In the case of PXC/PVP K12/SDS ternary GM, PXC on the particle surface may be amorphous; however, most of the PXC was in a
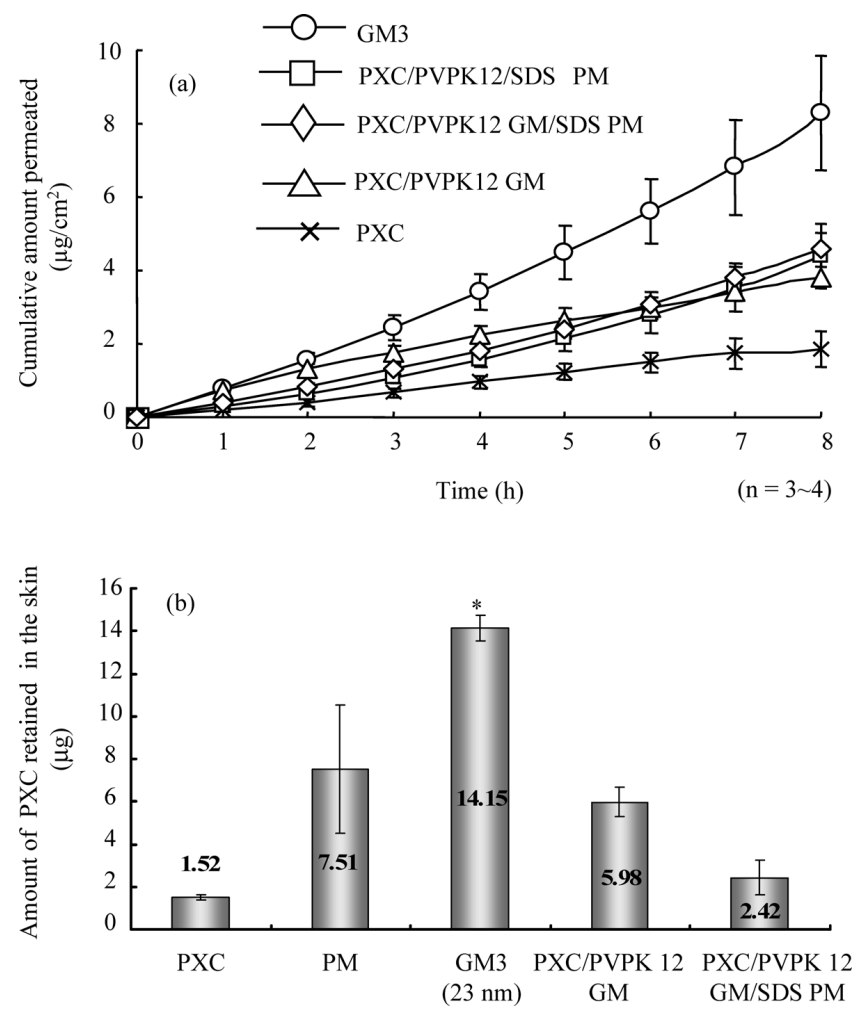

Fig. 4. Effect of Amorphous State on the Amount of (a) PXC Permeated and (b) Skin Retention

Each point represents the mean \pm S.D. of three to four experiments. $* p<0.01 v s$. PXC, PM, PXC/PVP K12 GM and PXC/PVP K12 GM/SDS PM. Statistical analysis was performed by ANOVA. 
Table 2. Effect of Amorphous PXC on the Skin Permeation Parameters of PXC

\begin{tabular}{lcl}
\hline \hline \multicolumn{1}{c}{ Sample } & $\begin{array}{c}\text { Cumulative amount permeated } \\
\text { for } 8 \mathrm{~h}\left(\mu \mathrm{g} / \mathrm{cm}^{2}\right)\end{array}$ & $\begin{array}{c}\text { Flux } \\
\left(\mu \mathrm{g} / \mathrm{cm}^{2} / \mathrm{h}\right)\end{array}$ \\
\hline PXC & $1.87 \pm 0.49$ & $0.25 \pm 0.06$ \\
PXC/PVPK12/SDS PM & $4.39 \pm 0.86^{a)}$ & $0.58 \pm 0.13^{a)}$ \\
GM3 $(23 \mathrm{~nm})$ & $8.31 \pm 1.56^{b, c)}$ & $1.07 \pm 0.21^{b, c)}$ \\
PXC/PVPK12GM & $3.81 \pm 0.29^{b)}$ & $0.52 \pm 0.05^{b)}$ \\
PXC/PVPK12 GM/SDS PM & $4.57 \pm 0.46^{b)}$ & $0.59 \pm 0.06^{b)}$
\end{tabular}

Each value represents the mean \pm S.D. of $3-4$ experiments. N.D.: Not detected, a) $p<0.05$ vs. PXC, b) $p<0.01$ vs. PXC, c) $p<0.01$ vs. PXC/PVPK12 GM and PXC/PVPK12 GM/SDS PM. Statistical analysis was performed by ANOVA.

nanocrystalline state. The effects of amorphous PXC on skin permeation and retention was examined using a $\mathrm{PXC} / \mathrm{PVP}$ K12 GM and SDS PM, abbreviated as PXC/PVP K12 GM/SDS PM. The skin permeation profiles and parameters of PXC are shown in Fig. 4a and Table 2, respectively. The cumulative permeated amounts of PXC in PXC/PVP $\mathrm{K} 12$ GM and PXC/PVP K12 GM/SDS PM for $8 \mathrm{~h}$ were significantly increased compared with those for unprocessed PXC. However, the amount of PXC permeated in both systems was still lower than the amount permeated in GM3. The flux of PXC in PXC/PVP K12 GM and PXC/PVP K12 GM/SDS $\mathrm{PM}$ was 0.52 and $0.59 \mu \mathrm{g} / \mathrm{cm}^{2} / \mathrm{h}$, respectively, almost the same as that seen with the PXC/PVP K12/SDS PM. In contrast, the flux of GM3 $\left(1.07 \mu \mathrm{g} / \mathrm{cm}^{2} / \mathrm{h}\right)$ was significantly higher than that of the PM. Skin retention of PXC in GM3 was also significantly higher than that of PXC/PVP K12 GM and PXC/PVP K12 GM/SDS PM, as shown in Fig. 4b. These results indicate that improved skin permeation and retention of GM3 is due to the size reduction of PXC nanoparticles, and not due to amorphization. Because the amorphous state of PXC in each formulation is not stable, it may crystallize in aqueous media. Since the GM3 suspension was transparent, it resembled a supersaturated solution of PXC. However, the GM3 suspension consisted of nanocrystalline PXC, and it formed a nanocrystalline suspension. Thus, it appears that skin permeation and retention behavior is related to PXC particle size.

In conclusion, size-dependent skin permeation and retention of PXC were observed when the particle size of the ternary GM was decreased to submicron order. Drug nanosuspensions with a size of less than $50 \mathrm{~nm}$, the size of which was reported by Shim et al., ${ }^{18)}$ Vogt et al., ${ }^{19)}$ and also shown in this study, appear to be useful for enhanced permeation. Amorphous PXC formed in the process of grinding did not affect skin permeation and retention. These findings are useful for understanding the skin permeation behavior of a drug in nanocrystalline suspension.

Acknowledgments This work was supported in part by a Grant-in-Aid from the Ministry of Education, Culture, Sports, Science and Technology (MEXT) of Japan (21590038) and by a Grant from the "High-Tech Research Center" and "Academic Frontier" Projects for Private Universities, with a matching fund subsidy from MEXT for 2007-2009.

\section{References}

1) Kreuter J., "Encyclopedia of Pharmaceutical Technology," ed. by Swarbrick J., Boylan J. C., Marcel Dekker, New York, 1994, pp. 165190.

2) Kawashima Y., Adv. Drug Deliv. Rev., 47, 1-2 (2001).

3) Merisko-Liversidge E., Liversidge G. G., Cooper E. R., Eur. J. Pharm. Sci., 18, 113-120 (2003).

4) Jinno J., Kamada N., Miyake M., Yamada K., Mukai T., Odomi M. Toguchi H., Liversidge G. G., Higaki K., Kimura T., J. Controlled Release, 111, 56-64 (2006).

5) Itoh K., Pongpeerapat A., Tozuka Y., Oguchi T., Yamamoto K., Chem. Pharm. Bull., 51, 171-174 (2003).

6) Pongpeerapat A., Itoh K., Tozuka Y., Moribe K., Oguchi T., Yamamoto K., J. Drug Del. Sci. Tech., 14, 441— 447 (2004).

7) Pongpeerapat A., Higashi K., Tozuka Y., Moribe K., Yamamoto K., Pharm. Res., 23, 2566-2574 (2006).

8) Shudo J., Pongpeerapat A., Wanawongthai C., Moribe K., Yamamoto K., Biol. Pharm. Bull., 31, 321-325 (2008).

9) Lee C. K., Uchida T., Kitagawa K., Yagi A., Kim N., Goto S., J. Pharm. Sci., 83, 562-565 (1994).

10) Ammara H. O., Ghorab M., El-Nahhas S. A., Kamela R., Int. J. Pharm., 327, 81-88 (2006).

11) Hammell D. C., Hamad M., Vaddi H. K., Crooks P. A., Stinchcomb A. L., J. Controlled Release, 97, 283-290 (2004).

12) Matsuda H., Arima H., Adv. Drug Deliv. Rev., 36, 81—99 (1999)

13) Ventura C. A., Tommasini S., Falcone A., Giannone I., Paolino D. Sdrafkakis V., Mondello M. R., Puglisi G., Int. J. Pharm., 314, 37-45 (2006).

14) Mássona M., Loftssona T., Másson G., Stefánsson E., J. Controlled Release, 59, 107-118 (1999).

15) Williams A. C., Barry B. W., Adv. Drug Deliv. Rev., 56, 603-618 (2004).

16) Suhonen T. M., Bouwstra J. A., Urtti A., J. Controlled Release, 59, 149-161 (1999).

17) Trommer H., Neubert R. H. H., Skin Pharmacol. Physiol., 19, 106121 (2006)

18) Shim J., Kang H. S., Park W., Han S., Kim J., Chang I., J. Controlled Release, 97, 477-484 (2004).

19) Vogt A., Combadiere B., Hadam S., Stieler K. M., Lademann J., Schaefer H., Autran B., Sterry W., Blume-Peytavi U., J. Invest. Dermatol., 126, 1316-1322 (2006).

20) Tsujimoto H., Hara K., Tsukada Y., Huang C. C., Kawashima Y., Arakaki M., Okayasu H., Mimura H., Miwa N., Bioorg. Med. Chem. Lett., 17, 4771-4777 (2007).

21) Lademann J., Richter H., Teichmann A., Otberg N., Blume-Peytavi U., Luengo J., Weisß B., Schaefer U. F., Lehr C., Wepf R., Sterry W., Eur. J. Pharm. Biopharm., 66, 159-164 (2007).

22) Han F., Li S., Yin R., Shi X., Jia Q., Drug Dev. Ind. Pharm., 34, 453458 (2008)

23) Piao H., Kamiya N., Hirata A., Fujii T., Goto M., Pharm. Res., 25, 896-901 (2008).

24) Mortensen L. J., Oberdörster G., Pentland A. P., DeLouise L. A., Nano Lett., 8, 2779-2787 (2008).

25) Okuyama H.,Ikeda Y., Kasai S., Imamori K., Takayama K., Nagai T., Int. J. Pharm., 186, 141-148 (1999).

26) Inoue K., Ogawa K., Okada J., Sugibayashi K., J. Controlled Release, 108, 306-318 (2005). 